\title{
AUTOMATIC SPEED VARIATION SYSTEM IN AUTOMOBILE LINKED WITH DOORS
}

\author{
B. Ramesh*1, Altrin Rijo ${ }^{2}$ \\ ${ }^{1}$ Professor, Mechanical (Mechatronics) Engineering Department, \\ Sri Muthukumaran Institute of Technology, chennai \\ ${ }^{2}$ Assistant Professor, General Engineering, Mechanical Department, \\ R.M.K College of Engineering and Technology, chennai. \\ rameshhcad@gmail.com ${ }^{1}$,rijo1151@gmail.com ${ }^{2}$
}

\begin{abstract}
:
The sudden accidents often occur when passengers traveling on the footpath of public transport at high speed. The sudden fall from footsteps at that speed may cause serious risk to passengers. In this paper, we present a passengers safety system that has two major features, one is to detect the passengers present in the footpath of the vehicle and the other one is to maintain the speed of the vehicle based on the detection of passengers in the footpath. The present work used IOT system that contains an ultrasonic sensor to detect passengers in a given area. Based on the sensor output of detection of passengers, the door of public transport will automatically open/close and also it will maintain or increase the speed of the bus based on the results. The development of this IOT system also implements an IEEE 802.11 technology to transmit the signal from public transport to mobile apps. The monitoring and controlling mobile application are developed which can receive/transmit the data from/to the controller of the system by using IEEE 802.11. This mobile application shows the status of detection of passengers, door status, and also the real-time speed of the public transport.
\end{abstract}

Keywords - Passenger safety, IoT, Monitoring system, Wireless Communication.

\section{Introduction}

Technological innovations have now begun to leap into the era of automation where present technologies are moving towards the Internet of Things (IoT) [1]. IoT is widely used in helping to develop safety in many areas [2] such as Medical, Machining Industries, Burglar detection, Transportation, etc. In public transportation, many accidents were happening all over the world due to neglect of closing doors in buses while moving. Most of the bus doors are design perspective, for the most part, passengers are versatile to enter and exit through them. However, passengers who are traveling on doorsteps are less aware of the speed vehicles approaching on the sideways of the bus and also not always easy to predict and avoid those accidents [3]. It is observed that hearing the accidents occurring due to the opening of the doorways in bus travel. 
The common object sensing technology in other research works, there is Camera, Infrared, radar, and so on [3]. Because the door is usually open while the vehicle is stationary. But while in our research it is difficult to wait for long until the passengers move to the safe place since it can cause unnecessary traffic behind the vehicle. So, when the passengers aboard the bus will start moving at a slow speed to avoid unnecessary traffic chaos and also to prevent any high-speed accidents the bus will move only at low speed until it makes sure all the passengers are moved to a safe position inside the bus.

Although the passengers standing near the door can be detected by using an integration of sensors, still the acceleration of the vehicle can cause sudden external force that impacts the passengers who are standing near the door path. Hence it is necessary to detect and control the acceleration of the vehicle moves, this can be detected by the accelerometer. When the acceleration is different from the normal situation or the acceleration crosses the threshold value, it is judges as a passenger's impact. This principle is the same as detecting whether the human body falls or not [4]. This can be estimated by (1)

$$
s=\sqrt{\left(x-x^{\prime}\right)^{2}+\left(y-y^{\prime}\right)^{2}+\left(z-z^{\prime}\right)^{2}}
$$

Where, $S$ indicates the strength of acceleration, $x, y$, and $z$ indicates the scale of axes, $x^{\prime}, y^{\prime}$ and $z$ ' indicates the previous scale of all axes.

\section{Literature Survey}

In article [6], tried to implement the idea of IoT on buses using temperature sensors (Thermistors) and Global Positioning System to detect the passengers and coordinates of the bus, the temperature in bus, etc, this method is reasonably good at detecting level but the main drawback is still the same as infrared i.e. when an object is not moving the sensor still recognize it as a passenger based on heat signature.

In article [7], implemented a method to detect passengers using suspension activities on board public transport vehicles. This method uses an elliptical head detection algorithm using in curvature profile of the human head as the cue. The main drawback in this method is that if the head is covered by cloth the geometric shape will not be detected.

Blind spot monitoring system which typically avoids accidents occurring near to the footsteps of the public transport vehicle. This type of system uses the camera to monitors the driving path of the vehicle to check for vehicles within the blind spot area. This system is designed to neglect the accidents occurring between two vehicles and also accidents occurring to passengers traveling in footpath by another vehicle. The limitation of this system is that it is a very tedious process in an Image processing system to remove the background area while the vehicle traveling at high speed. This requires a high-speed processing device.

The use of Ultrasonic sensors in the detection of passengers is the main focus here, the use of Ultrasonic sensors can neglect the limitation of Camera, Infrared to detect the 
presence of passenger because it can only detect the presence of an object, not the heat signature, hence it can only detect the object presence and its distance of the object detected. Ultrasonic uses sound waves that hit the object and reflect the receiver, the time duration of the transmission and receiver can be used to calculate the presence of an object and its distance.

\section{Methodology}

The methodology of this approach has four stages namely Requirements, Planning, Selection of Material, and Design.

\subsection{Requirement}

This system requires both the Mechanical and Electronics parts to work together. The system requirements include the design of the frame, sensors to identify the passengers, the Speed of the vehicle, the Status of the door, and a controller to send/ receive signals in and out of all these electronic devices. All these devices can be monitored and controlled by using IEEE 802.11 module. Requirements for this device include:

1. The board used is ESP $8266 \mathrm{Wi}-\mathrm{Fi}$ SoC as a wireless module to monitor and control data from/to the device.

2. The controller Arduino IDE connects all the electronic devices that serve as a medium for controlling all the devices by programming.

3. The Ultrasonic sensor is a trigger to identify the passengers standing near the designated area.

4. Jumper cables to connect all the modules.

5. Breadboard to assemble all devices.

\subsection{Planning}

The planning stage, such as finalizing the language to be used for programming to control devices that will be used in the development of the system.

All the devices are integrated using Arduino IDE using Embedded $\mathrm{C}$ programming language, which is supported by ESP8266 library as support for controlling the device wirelessly that is easily monitored by mobile applications. The monitoring applications are designed using Arduino Studio as a compiler supported by JAVA programming.

\subsection{Selection of material}

The material is the most important thing in hardware design that plays a vital role in displaying the idea in the prototype of the real-life application.

Mild steel is an iron that contains $0.05-0.25 \%$ of the carbon to achieve high malleable and ductile properties. It is relatively low tensile strength but by carburizing the mild steel can increase its surface hardness.

\subsection{Design}

The design is made that can explain the flow of the system. The Internet of Things involves both hardware and software. The system is designed to detect the passengers on the bus that can be monitored using a mobile device. The flow chart of the model is presented in Figure 1 and also design of the Frame is presented in Figure 2. 


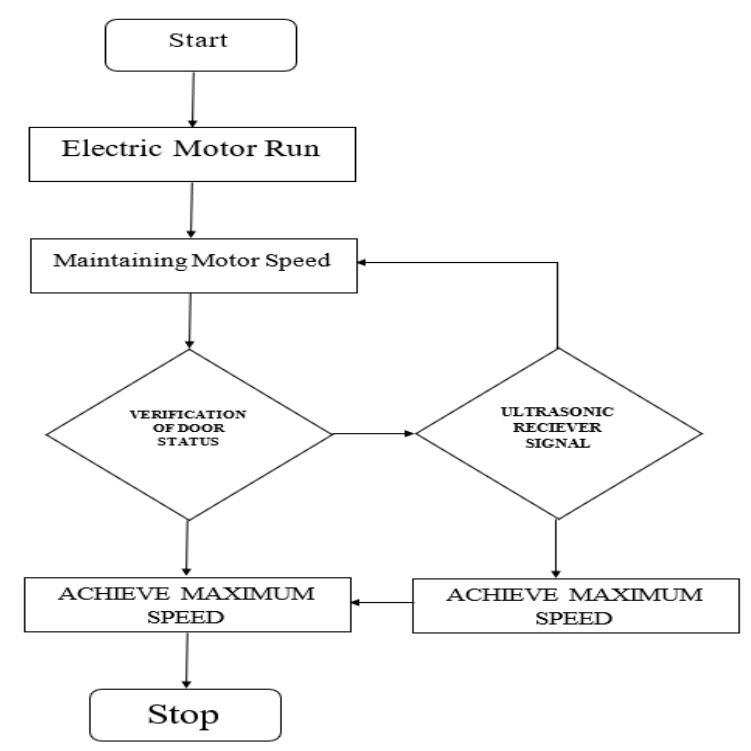

Figure. 1 Flowchart Diagram

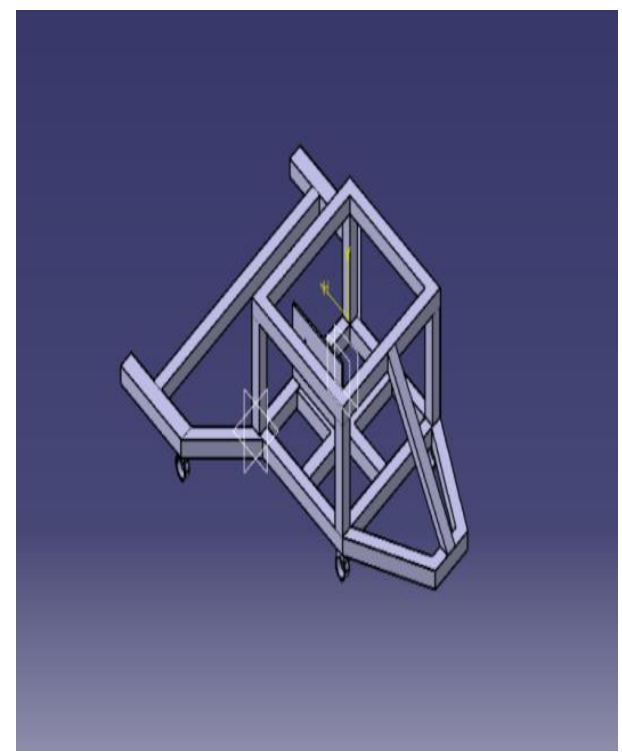

Figure. 2 Design of the Mechanical frame

\section{Experimental Result}

\subsection{Mathematical Modelling}

The Simulink model is designed and constructed based on the motor parameters.

Figure 3 is the Mathematical modelling for motor control.

This mathematical modelling consists of a 24V DC motor at Speed of 1750RPM which is controlled by the $4 \mathrm{H}$-Bridge circuit. In this DC motor, the supply is given to the field connections so that it can be excited separately either by using a shunt connection or series connection. The parameters of the motors are.

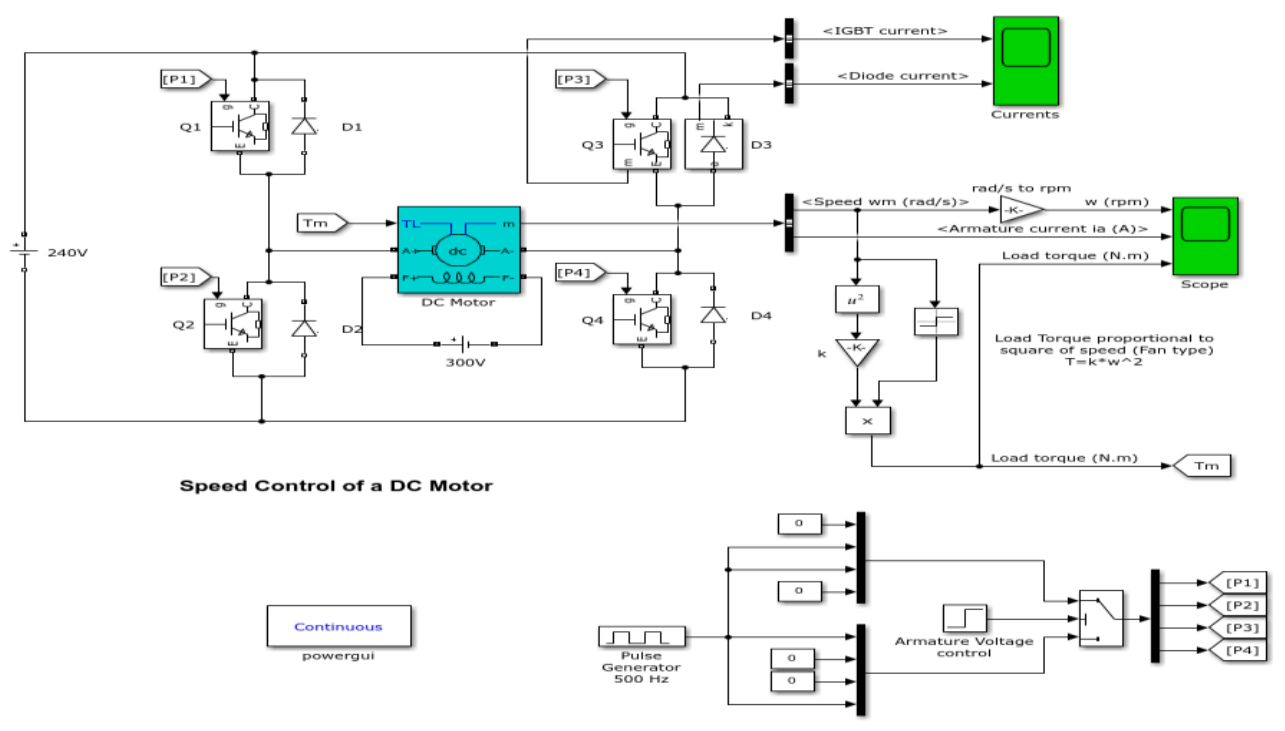

Figure. 3 Mathematical modelling based on motor parameters 
Armature resistance and inductance $[\mathrm{Ra}(\Omega) \mathrm{La}(\mathrm{H})]$

$=2.581 \Omega, 0.028 \mathrm{H}$

Field resistance and inductance $[\operatorname{Rf}(\Omega) \operatorname{Lf}(\mathrm{H})]$

$=281.3 \Omega, 156 \mathrm{H}$

Field-armature mutual inductance Laf $(\mathrm{H})$

Total inertia $\mathrm{J}\left(\mathrm{kg} \cdot \mathrm{m}^{2}\right)$

$=0.9483 \mathrm{H}$

Viscous friction coefficient Bm (N.m.s)

$=0.02215 \mathrm{~kg} \cdot \mathrm{m}^{2}$

Coulomb friction torque Tf (N.m)

$=0.002953 \mathrm{Nms}$

$=0.516 \mathrm{Nm}$

\subsection{Graphical User Interface}

The overall system consists of a Graphical user interface, WIFI module, Controller, Motor Driver, Motor, Ultrasonic Sensor. Figure 4 shows the overall System Process.

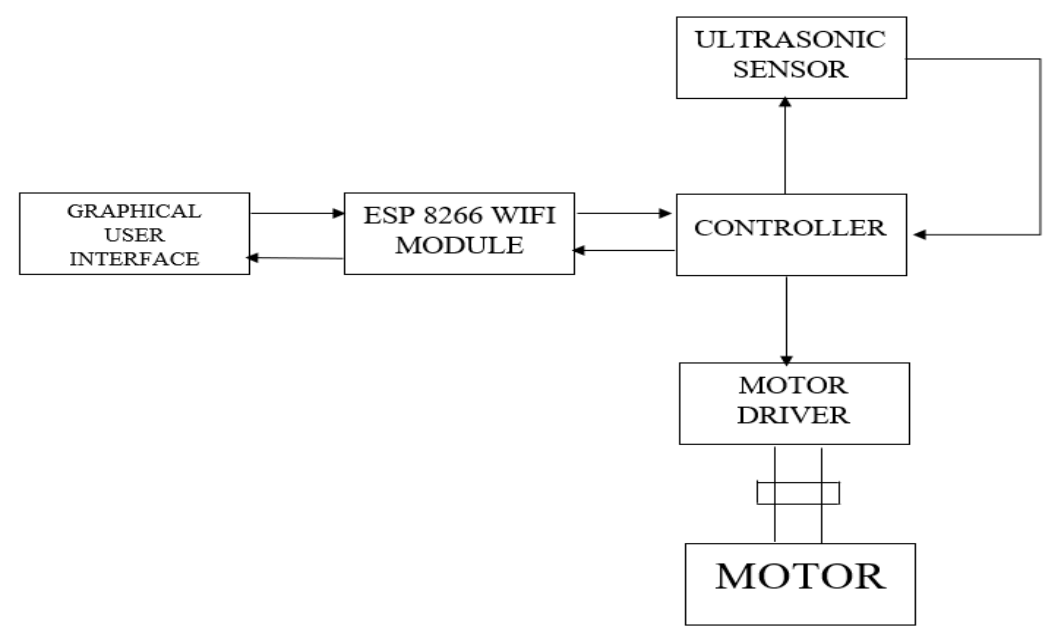

Figure. 4 Overall System process

The Implementation of this system has two software programming, Arduino IDE programming for the devices to embedded into a single controller and for monitoring purpose Arduino Studio application has been used to develop a Graphical user interface to control and monitor the system remotely. Figure 5 shows the Graphical user interface of the system.

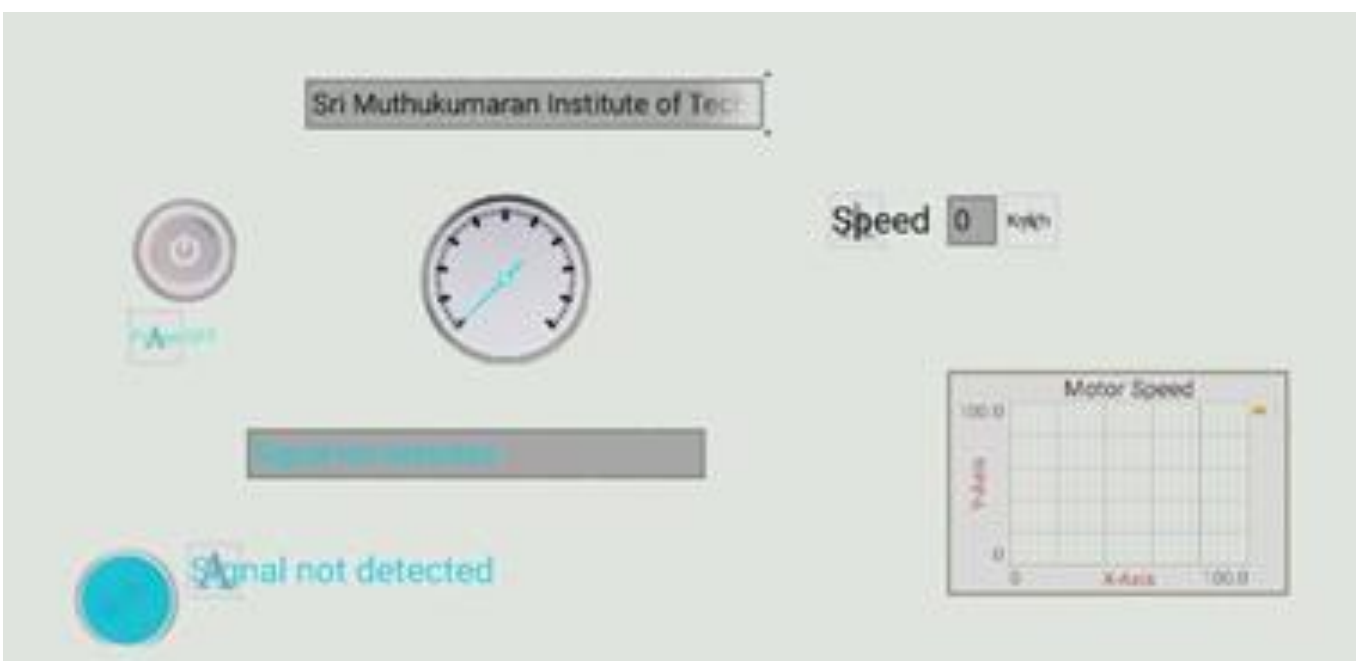

Figure. 5 Graphical User Interface of the system 
The Graphical User Interface (GUI) designed in such a way to both control and monitor the system, the power button controls the overall system power and also the GUI contains a speedometer to display the current speed, Ultrasonic obstacle detection, Door Signal, and a Graphical display of the speed of the motor.

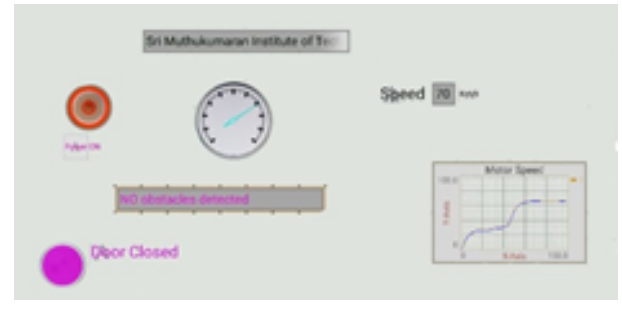

Figure. 6 Obstacle detection

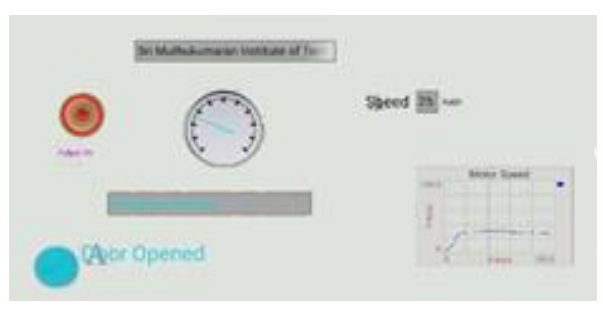

Figure. 7 No obstacle detection

The Figure $6 \& 7$ represents the two conditions of the system reacts when there is an obstacle and No obstacle detection. After turning on the system when the obstacle is present in the designated area i.e in the staircase of the public transportation and the door will maintain its open state, then the vehicle will travel at the minimum speed of $25 \mathrm{Km} / \mathrm{hr}$. When there is no obstacle is present in the staircase of the vehicle then the door will automatically close and the vehicle starts to accelerate gradually from $25 \mathrm{Km} / \mathrm{hr}$ to $70 \mathrm{Km} / \mathrm{Hr}$ max speed.

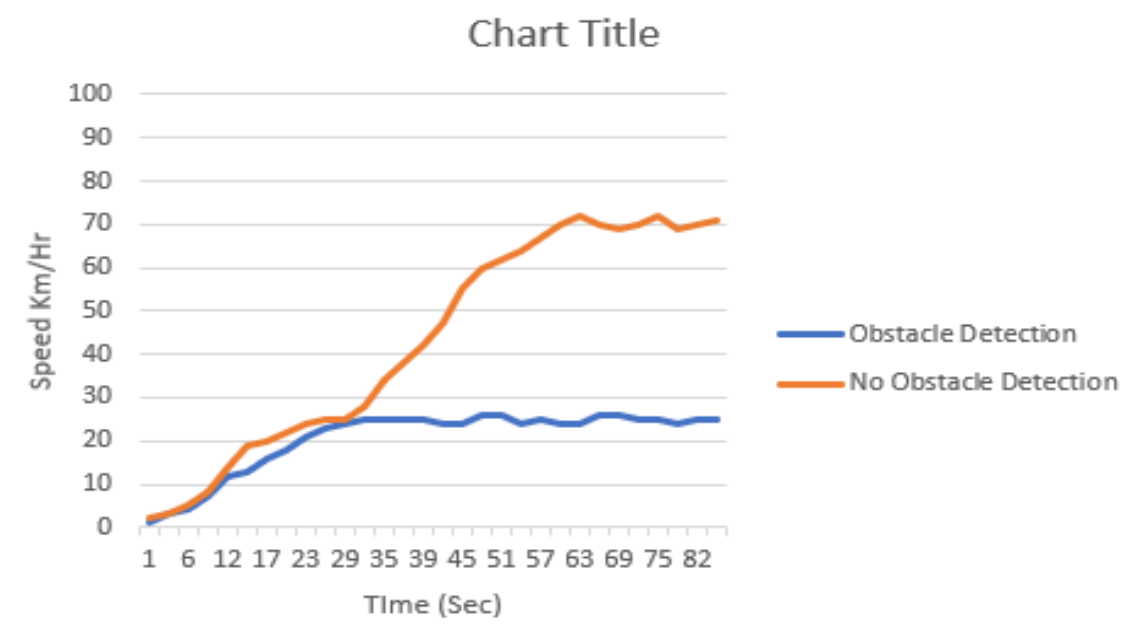

\section{Figure. 8 Comparison of the speed in two different conditions}

Figure 8 shows the output response of the system in two different conditions. The Blue plot represents the speed of the vehicle when an obstacle is present and also the door is opened which maintains the low speed. The orange plot represents the Speed of the vehicle when there is no obstacle and also the door is closed, in which the speed gradually increases to its maximum value.

\section{Conclusion}

This paper presents the IOT-based detection and monitoring of passengers' safety while traveling in the footpath of public transport. The ultrasonic sensor used here has detected the passengers' presence in the footpath with an accuracy of $100 \%$ from the results of multiple tests have taken. In addition to that, the IEEE 802.11 WIFI module 
has received and transmitted the data in real-time from the controller to the mobile application as well. The controller which embedded all the electronic components has received/ send data from/to the mobile application to all the electronics devices.

After this experimental study, It is found out that this controller is suitable for the prototype model and for the real-time system it is required for clock frequency controller to process at a faster rate. Multiple ultrasonic sensors at a different orientation to get more accurate detection as well.

\section{References}

[1] F. M. S. Nursuwars and A. Rahmatulloh, "RFID for nurse activity monitoring in the hospital's nurse call system with Internet of Thing (IoT) concept," in IOP Conference Series: Materials Science and Engineering, vol. 550, no. 1. (2019), pp. 1-7

[2] A. Karia, L. V. Budhwani, and V. S. Badgujar, "IoT-Key Towards Automation," 2018 International Conference on Smart City and Emerging Technology, (2018), pp. 1-5

[3] Bourke AK van de Ven P, Gamble M, O'Connor R, Murphy K, Bogan E, McQuade E, Finucane $P$, Olaighin $G$, Nelson J., "Evaluation of waist mounted tri-axial accelerometer-based fall-detection algorithms during scripted and continuous unscripted activities", Journal of Biomechanics,( 2010), pp. 3051-3057

[4] Élodie Morin, Mickael Maman, Roberto Guizzetti, Andrzej Duda, "Comparison of the Device Lifetime in Wireless Networks for the Internet of Things", IEEE Access, Vol. 5, (2017), pp. 7097-7114

[5] S. Birchfield. Elliptical head tracking using intensity gradients and color histograms. "In IEEE Conference on Computer Vision and Pattern Recognition”, Santa Barbara, California, (1998)

[6] N. D. Bird, O. Masoud, N. P. Papanikolopoulos, and A. Isaacs. Detection of loitering individuals in public transportation areas. IEEE Transactions on Intelligent Transportation Systems, Vol.6, no2, (2005), pp.167-177 